\title{
Value of imprint cytology for ultrasound-guided transthoracic core biopsy
}

\author{
W-Y. Liao*, J-S. Jerng*, K-Y. Chen*, Y-L. Chang\#, P-C. Yang*, S-H. Kuo
}

Value of imprint cytology for ultrasound-guided transthoracic core biopsy. W-Y. Liao, J-S. Jerng, K-Y. Chen, Y-L. Chang, P-C. Yang, S-H. Kuo. (C)ERS Journals Ltd 2004. ABSTRACT: The aim of this study was to investigate the possible additional diagnostic information provided by imprint cytology when performing ultrasound-guided transthoracic core biopsy and to evaluate whether it could optimise the biopsy procedure.

A total of 155 transthoracic core biopsies with touch imprint smears were performed under ultrasound guidance, with 127 malignant and 28 benign lesions. The imprint smears were stained using Riu's method and interpreted by a cytopathologist. These were compared with the histopathology of core biopsy specimens and the final diagnosis of malignant versus benign disease.

The overall diagnostic accuracy of imprint cytology was 94\% (146 out of 155 ). Histopathological analysis showed an overall accuracy of $94 \%(146$ out of 155), with a sensitivity of 94\% (119 out of 127) and negative predictive value of 79\% (27 out of 34). The combination of these two methodologies had an increased overall accuracy and negative predictive value of $98 \%(152$ out of 155$)$ and $90 \%(28$ out of 31$)$, respectively. The results of imprint cytology and histopathology were in agreement in 143 patients $(92 \%)$.

In conclusion, imprint cytology of ultrasound-guided transthoracic core biopsy is a sensitive procedure for diagnosing peripheral thoracic lesions, and it may increase the diagnostic accuracy and cancer negative prediction of biopsy alone. With an on-site approach, imprint cytology may help to assess the adequacy of biopsy specimens and optimise the biopsy procedure.

Eur Respir J 2004; 24: 905-909.
Depts of *Internal Medicine, ${ }^{\#}$ Pathology and Laboratory Medicine, National Taiwan University Hospital and National Taiwan University College of Medicine, Taipei, Taiwan.

Correspondence: S-H. Kuo, Dept of Laboratory Medicine, National Taiwan University Hospital and National Taiwan University College of Medicine, 7 Chung-Shan S. Road, Taipei 100, Taiwan.

Fax: 886223932056

E-mail: shkuo@ha.mc.ntu.edu.tw

Keywords: Core biopsy

imprint cytology

thorax

ultrasound

Received: March 112004

Accepted after revision: August 172004
Image-guided transthoracic core biopsy, using fluoroscopic, computed tomographic (CT) or ultrasonographic guidance, is a well-established and safe method for diagnosing malignant and benign thoracic lesions [1-5]. Despite the high diagnostic accuracy of transthoracic core biopsy, specimens inadequate for histological analysis have been encountered in up to $15 \%$ of grossly adequate specimens [6]. To improve the diagnostic yield, several authors have reported the usefulness of frozen section for immediate pathological diagnosis [6, 7]. However, it takes $15-20 \mathrm{~min}$ to obtain a pathological diagnosis for a frozen section and much longer if the initial sampling is unsatisfactory.

Intra-operative cytology of touch preparations has been used as an alternative to frozen section since it was first reported in 1927 [8-13]. This method has been utilised to obtain immediate cytological diagnosis for core needle and bronchoscopic forcep biopsies [14-16]. As an adjunct to the histopathology of core biopsy specimens, imprint cytology helps to guarantee that the specimens obtained adequately represent the lesion.

Ultrasonography is as effective as CT for guidance of transthoracic biopsies of peripheral pulmonary lesions and mediastinal tumours, and it offers a number of advantages [5, 17, 18], including bedside approach, lower cost and no radiation exposure. In this study, the diagnostic accuracy of imprint cytology for ultrasound-guided transthoracic core biopsy was retrospectively evaluated, and the concurrency between cytological and histological results was assessed to verify its role in the conduct of biopsy procedures.

\section{Materials and methods}

From January 1999 to December 2000, ultrasound-guided transthoracic core biopsies for peripheral thoracic lesions were performed in 240 patients at the National Taiwan University Hospital (Taipei, Taiwan). In addition, imprint cytology of the biopsy specimens was obtained in 163 out of the 240 patients.

Transthoracic core biopsy was carried out with an 18-gauge Urocut needle through a sterile puncture transducer (Aloka UST-507 BP; Aloka Co. Ltd, Tokyo, Japan), as described previously [5]. The biopsy specimen was directly imprinted on a slide with light touch to avoid gliding movement and was stained using Riu's method after being air-dried [19]. The biopsy specimen was then put into a formaldehyde solution (10\% formalin) for histological examination. The imprint smear was checked on-site by a pulmonologist, who performed the procedure and then decided whether an additional biopsy was needed.

All imprint smears were reviewed by a board-certified cytopathologist who was not aware of the histopathological results of the specimens or of the final diagnoses of all the patients. Cytological diagnoses were classified into one of four 
categories: inadequate specimen; negative for malignancy; suspicious for malignancy; or positive for malignancy. Inadequate specimens were defined as $<100$ lung cells (alveolar macrophages and/or pulmonary epithelial cells) on a smear without neoplastic cells. Negative for malignancy indicated that there were no malignant cells when the specimen was adequate. Imprint cytology without a clearcut malignant process but with some abnormal feature was classified as suspicious for malignancy. A specific benign diagnosis (granulomatous inflammation, an identifiable microorganism or a specific benign tumour) was suggestive of a negative result and the percentages of various cells (epithelial cells, macrophages, lymphocytes and neutrophils) were recorded if a non-specific benign diagnosis (chronic inflammation, an inflammatory cell aggregate or necrosis) was encountered.

The definite diagnosis of malignant thoracic lesions was based on: 1) the histopathological analysis of the surgical specimen; or 2) the histopathological analysis of the cutting biopsy specimen and a subsequent clinical course showing progressive disease and/or metastatic disease that was consistent with cancer. The definite diagnosis of benign thoracic lesions was based on: 1) surgical confirmation; 2) subsequent disappearance of the lesion or decrease in its size; or 3) followup chest radiographs or CT scans showing that the lesion remained stable for at least 2 yrs.

A true-positive result for malignancy indicated that imprint cytology or histopathological analysis of the biopsy specimen was diagnostic of a malignant thoracic lesion. A suspicion of malignancy for imprint cytology was considered as truepositive if the final diagnosis was malignancy and as falsepositive if the final diagnosis was benignity. A false-negative result for imprint cytology was considered when imprint cytology of the biopsy specimen showed no malignancy but the final diagnosis was malignant. Conversely, a false-negative result for core biopsy indicated that histopathological analysis of the biopsy specimen showed the thoracic lesion to be benign but surgery or the subsequent clinical course showed the lesion to be malignant.

The results of cytological analysis of imprint smears and histopathological analysis of biopsy specimens were compared, and the accuracy, sensitivity, specificity, and positive and negative predictive values of these two methods were evaluated both separately and combined. If either the histopathology or imprint smear showed true-positive, it was counted as true-positive when analysed in combination. The number of biopsy procedures (equivalent to needle passes to obtain a positive biopsy) and complications of the biopsy procedure were recorded for all patients.

Data regarding number of biopsy procedures were expressed as mean $\pm \mathrm{SD}$. The unpaired t-test was used to compare the number of biopsy procedures between groups and the Chi-squared test was used to compare qualitative variables. A p-value of $<0.05$ was considered statistically significant.

\section{Results}

Out of the 163 patients evaluated, a final diagnosis was unavailable in eight patients, resulting in a study population of 155 . The study population consisted of 104 males and 51 females, with a mean age of 60 yrs (range 15-86). Out of the 155 lesions subjected to biopsy, 131 were lung parenchymal lesions, four were chest wall lesions and 20 were mediastinal lesions. A total of 127 lesions (127 out of $155,82 \%$ ) were malignant and 28 were benign ( 28 out of $155,18 \%$ ), according to the final diagnoses. Imprint smears were able to be interpreted in 151 out of $155(97 \%)$ patients and 153 out of $155(99 \%)$ biopsy specimens had sufficient material for histopathological analysis. Cytological diagnosis of imprint smears yielded the correct diagnosis of malignant versus benign in 146 out of $155(94 \%)$ thoracic lesions. Histopathological analysis of biopsy specimens showed an accuracy of $94 \%$ (146 out of 155). The combined overall accuracy was $98 \%$ (152 out of 155 ) (table 1). The results of imprint cytology and histopathology of biopsy specimens were in agreement in 143 patients $(92 \%)$.

\section{Malignant thoracic lesions}

There were 127 patients who had a final diagnosis of malignancy. Twenty-seven out of the 127 lesions were confirmed on the basis of the histology of the surgical specimen, while the remaining 100 malignant thoracic lesions were diagnosed on the basis of histopathological evaluation of the biopsy specimen and subsequent clinical course. Among the 127 malignant thoracic lesions there were 77 non-small cell lung cancers, 15 small cell lung cancers, one pulmonary leiomyosarcoma, one atypical carcinoid tumour, eight lymphomas, four thymic carcinomas, four malignant germ cell tumours and 17 metastatic cancers.

The cytological diagnosis of imprint smears for these 127 malignant thoracic lesions was malignancy in 118 patients, suspicious in four patients and negative in five patients. Histopathological analysis of biopsy specimens showed malignancy in 119 patients, was negative in seven patients and inadequate in one. Thus, the sensitivities for imprint cytology and histopathological analysis of biopsy specimens were $96 \%$ (122 out of 127) and 94\% (119 out of 127), respectively. The combination sensitivity was $98 \%$ (124 out of 127). The relationship between the results of these two diagnostic methods is demonstrated in table 2. Imprint cytology and histopathology of biopsy specimens were in agreement in 120 out of 127 patients $(94 \%)$. Out of the 119 biopsy specimens with true-positive histopathological results,

Table 1.-Results of imprint cytology and histopathology of biopsy specimens according to the final diagnosis

\begin{tabular}{lcccccccc}
\hline & \multicolumn{3}{c}{ Benign } & & \multicolumn{4}{c}{ Malignant } \\
\cline { 2 - 4 } \cline { 6 - 8 } & SB & NB & IS & & TP & S & FN & IS \\
\hline Imprint cytology & 3 & 21 & 4 & & 118 & 4 & 5 & 0 \\
Biopsy & 14 & 13 & 1 & & 119 & 0 & 7 & 1
\end{tabular}

SB: specific benign; NB: non-specific benign; IS: inadequate specimen; TP: true-positive; S: suspicious; FN: false-negative. Benign: $n=28$; malignant: $\mathrm{n}=127$.

Table 2. - Relationship between the results of imprint cytology and histopathology of biopsy specimens for 127 malignant thoracic lesions

\begin{tabular}{lcccc}
\hline & \multicolumn{3}{c}{ Imprint cytology } & Total \\
\cline { 2 - 4 } & TP & S & FN & \\
\hline Core biopsy & & & & \\
TP & 113 & 4 & 2 & 119 \\
FN & 4 & 0 & 3 & 7 \\
IS & 1 & 0 & 0 & 1 \\
Total & 118 & 4 & 5 & 127 \\
\hline
\end{tabular}

TP: true-positive; S: suspicious; FN: false-negative; IS: inadequate specimen. 
imprint smears of these specimens were malignant or suspicious in $117(98 \%)$. The other two thoracic lesions, one small cell lung cancer and one non-small cell lung cancer, had negative imprint cytology. Five patients with negative histopathology results or inadequate specimen but positive imprint cytology were proven to be non-small cell lung cancer by surgery (two), bronchial biopsy (one) or pleural effusion cytology (two). Two out of the three lesions with falsenegative histopathology and imprint cytology showed predominant pulmonary epithelial cells (99 and 100\%) in imprint smears. Figure 1 demonstrates the histopathology and imprint smear of a biopsy specimen taken from a $69-\mathrm{yr}-$ old female with a diagnosis of lung cancer.

\section{Benign thoracic lesions}

The final diagnosis was benign in 28 patients. The final diagnosis was obtained by histological examination of the surgical specimen in 11 patients (one tuberculosis, two organising pneumonia, one localised fibrous tumour, one sclerosing haemangioma, one desmoid tumour, two thymoma, one mediastinal lipoma, one mature teratoma and one fibrous dysplasia) and by clinical follow-up in the remaining 17 (nine tuberculosis, five lung abscess, one organising pneumonia, one amyloidosis and one neurogenic tumour).

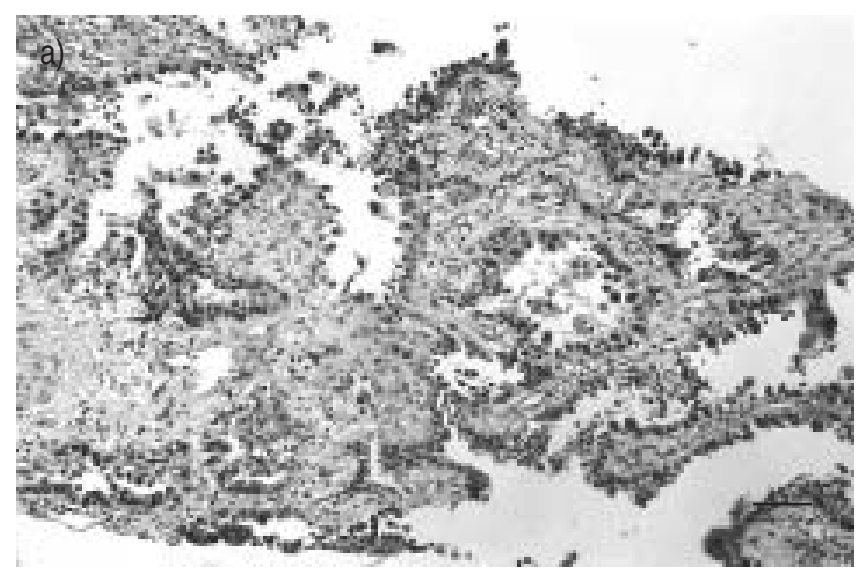

b)

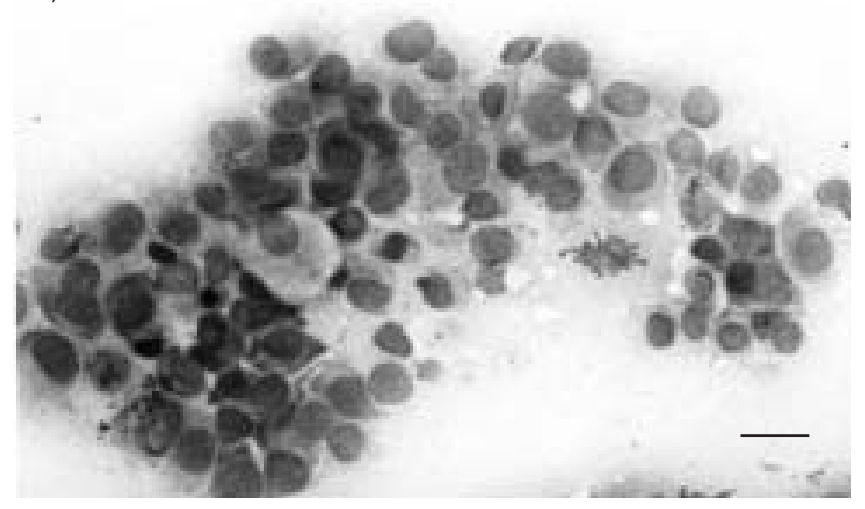

Fig. 1.-a) Histopathology of ultrasound-guided transthoracic biopsy specimen from a 69-yr-old female shows well-differentiated adenocarcinoma composed of bronchioloalveolar and acinar patterns (haematoxylin and eosin). b) Imprint smear of the same specimen reveals clusters of adenocarcinoma cells with eccentric nuclei and indistinct nucleoli (Riu's stain). Scale bars $=100$ and $25 \mu \mathrm{m}$, for a and $\mathrm{b}$, respectively.
The median follow-up period for the 17 patients was 12 months (range 5-30) and the lesions regressed during the follow-up period in 15 out of the 17 patients. One patient with a diagnosis of amyloidosis by histopathology of biopsy specimen was proven to be systemic amyloidosis in the subsequent course (intestine and lymph nodes involvement). Another patient diagnosed as neurogenic tumour by histopathology of biopsy specimen refused surgical resection, although the disease remained stable in the 24-month followup period.

Cytological analysis of imprint smears in the 28 benign patients obtained a specific benign diagnosis in three (two thymomas, one neurogenic tumour), non-specific benign diagnosis in 21 and inadequate specimen in four. Histopathological analysis of biopsy specimens obtained a specific benign diagnosis in $14(50 \%)$, a non-specific benign diagnosis in 13 and an inadequate specimen in one patient. Out of the four patients with inadequate specimens for cytological interpretation, one had fibrous dysplasia, one with localised fibrous tumour, one with mediastinal lipoma and one with tuberculosis. One lesion with an inadequate specimen for histopathological diagnosis was proven to be a mature teratoma by surgery. Out of the 21 patients whose imprint cytology showed a non-specific benign diagnosis, the percentages of pulmonary epithelial cells on the imprint smears ranged $0-79 \%$, with a mean of $24 \%$. The negative predictive values for imprint cytology and histopathology of biopsy specimens were $83 \%$ (24 out of 29 ) and $79 \%$ (27 out of 34), respectively. The negative predictive value increased to $90 \%$ (28 out of 31 ) with the two procedures combined. There were no falsepositive results.

\section{Number of biopsy procedures and complications}

The mean number of biopsy procedures in these 155 patients was $3.1 \pm 1.5$ (range 1-8). When all patients were divided into two groups, one had a positive or suspicious cytological diagnosis $(n=33)$ and the other had a negative or inadequate cytological diagnosis $(n=122)$; the mean number of biopsy procedures were $3.7 \pm 1.8$ and $2.9 \pm 1.3$, respectively $(p=0.03)$. The relationship between the number of biopsy procedures and final diagnosis is depicted in table 3 . Ten patients ( 10 out of $28 ; 35.7 \%$ ) with benign diagnosis had more than four biopsy procedures as compared with 16 (16 out of $127 ; 12.6 \%)$ in patients with malignant diagnosis $(\mathrm{p}=0.003)$. Complications were encountered in four out of the 155 patients $(2.6 \%)$ : two had haemoptysis and the other two had pneumothorax. There was no requirement for chest tube drainage or transfusion in these four patients. The mean number of biopsies in these four patients was $2.3 \pm 1.5$ (range $1-4)$.

Table 3. - Relationship between number of biopsy procedures and final diagnosis

\begin{tabular}{lcr}
\hline & Malignant & Benign \\
\hline $\begin{array}{lcc}\text { Subjects n } \\
\text { Biopsy procedures n (\%) }\end{array}$ & 127 & 28 \\
1 & $11(8.7)$ & $4(14.3)$ \\
2 & $43(33.9)$ & $4(14.3)$ \\
3 & $39(30.7)$ & $4(14.3)$ \\
4 & $18(14.2)$ & $6(21.4)$ \\
$\geqslant 5$ & $16(12.6)$ & $10(35.7)$ \\
\hline
\end{tabular}




\section{Discussion}

The results presented here demonstrate that imprint cytology for ultrasound-guided transthoracic core biopsy is a sensitive method for diagnosing malignant thoracic lesions $(96 \%)$ and can increase cancer negative prediction by integrating the cytological result with histopathology (90 versus histopathology alone $79 \%$ ). This study also shows that imprint cytology can predict the malignant nature of core biopsy specimens (98\%). Predominant epithelial cells (>99\%) on imprint smears suggest a high probability of false-negative histopathology as compared with less $(<80 \%)$ pulmonary epithelial cells (two out of two, $100 \%$ versus one out of $22,5 \%$ ) on imprint smears with non-specific benign diagnosis.

Although image-guided transthoracic fine-needle aspiration biopsy has a high diagnostic yield for malignant lesions, transthoracic core biopsy has been reported to be superior to needle aspiration for specific diagnosis of benign thoracic lesions and mediastinal tumours, determining cancer cell type and cancer-negative prediction [1-7]. Interventional radiologists are sometimes not sufficiently confident that the tissue cores obtained are adequate by gross inspection and that the histopathological diagnosis is correct. The reported inadequate specimen for image-guided transthoracic core biopsy has ranged $0-15 \%[1-7]$. To further improve the diagnostic accuracy of transthoracic core biopsy, SAKAI et al. [6] reported the usefulness of frozen section histopathological diagnosis for transthoracic core biopsy. In their series, sufficient material was obtained in 47 out of $55(85 \%)$ patients and a second biopsy for frozen section was needed in seven out of $47(15 \%)$ lesions. The first specimens from seven lesions that required second biopsy were grossly adequate, while the eight lesions with insufficient material were small $(<2 \mathrm{~cm})$ and had final diagnosis by cytological examinations. HAYASHI et al. [7] previously reported 52 lesions using the same technique and gained a similar result. In their series, three out of the 47 lesions with sufficient material required a second biopsy, while three out of the five lesions with insufficient material due to limited specimen had final diagnosis by cytological examinations. Despite the high efficacy of frozen section histopathology for transthoracic core biopsy, the use of this technique may be discouraging because it is time consuming and has a high failure rate.

In this study, imprint cytology was used to assess the adequacy of the biopsy specimen. There were only two patients (two out of $155,1 \%$ ) with inadequate specimens. Out of the 119 biopsy specimens with true-positive histopathological results, imprint smears of these specimens were positive or suspicious in $117(98 \%)$ cases. The other five patients who had positive imprint cytology results and false-negative or inadequate specimens of core biopsies were proven to be malignant by surgery or clinical course. Thus, positive imprint cytology results indicate true-positive and a further biopsy attempt is not necessary. Conversely, if an imprint smear shows no malignant cells and predominant pulmonary epithelial cells, further biopsy or diagnostic evaluation should be considered because of the high probability of false-negative histopathology. This high false-negative rate may be due to sampling of the adjacent normal lung tissue, which represents predominant pulmonary epithelial cells on imprint smears. Although the results of this study show that lesions with suspicious imprint cytology were finally diagnosed as malignancy, this should be interpreted cautiously and further procedures for definitive diagnosis are indicated when suspicious imprint cytology is encountered.

Since the introduction of imprint cytology for surgical pathology by DUDGEON and PATRICK [8] in 1927, this technique has been widely accepted as an adjunct to frozen section histopathology for intra-operative diagnosis [9-13]. With the exception of intra-operative diagnosis, imprint cytology has been used to obtain a rapid diagnosis for intra-abdominal lesions [14] and core biopsy of breast, as well as bronchoscopic forcep biopsies. AlBERT et al. [15] have previously reported an overall diagnostic accuracy of $95.4 \%$ for core needle biopsy of breast lesions. Popp et al. [16] used imprint cytology for bronchoscopic forcep biopsies and obtained a superior sensitivity as compared with histopathological results (84.9 versus $62.3 \%$ ). In the current series, imprint cytology of core biopsy specimens had an overall diagnostic accuracy of $94 \%$, which was comparable to histopathological results of biopsy specimens (94\%), and increased to $98 \%$ when combined with histopathology.

In addition to its comparable accuracy to frozen section histopathology, imprint cytology has been found to be more advantageous, because it is less costly and less time consuming [10]. Riu's stain, modified from Wright's stain, was first introduced for blood smear staining in 1956 and has been widely used for varied cytological examinations [20]. It takes only $90 \mathrm{~s}$ to complete a staining procedure with Riu's method and the diagnostic accuracy is equal to that achieved with Papanicolaou's stain when diagnosing pulmonary malignancies [21]. In the series presented here, Riu's method was used to stain imprint smears immediately after biopsy so that tentative diagnoses could be obtained rapidly. This determined whether further biopsy was needed and facilitated subsequent management while waiting for the histopathological results.

Moreover, several authors have reported that imprint cytology has superior quality to frozen section histopathology, especially for small specimens where there is a sampling error and potential loss of cryosectioning of lesional tissue, which is necessary for a permanent histopathological diagnosis [9-12]. In this series, four lesions with false-negative histopathology and one lesion with inadequate specimen were accurately diagnosed as malignant by imprint cytology. The possible cause of this discrepancy may be due to the small specimen or because there were only small areas of malignant cells within the specimens. The malignant component of a core biopsy specimen may be lost during the processing of permanent section, but imprint smear preserves the malignant cells regardless of the small specimen.

Despite the high accuracy of imprint cytology, frozen section and permanent histopathology should not be replaced, as cytologically well-differentiated tumours and tumours with a dense fibrous stroma and fat tissue cannot be diagnosed using this method [9]. In the current series, inadequate specimens of imprint smears were encountered in four benign lesions. Three of them had dense fibrous stroma and one was a lipoma. In addition, only three out of $28(11 \%)$ lesions obtained specific benign diagnoses by imprint cytology as compared with 14 out of $28(50 \%)$ by histopathology.

Since the co-axial technique was not used in this study, multiple pleural passes were required to obtain as many biopsy specimens as possible. Previous studies investigating complications of transthoracic biopsy have demonstrated that lesion size, emphysema, age and needle size, but not number of needle passes, affect the risk of pneumothorax [22, 23]. This study also showed no correlation between complications and number of biopsy procedures. However, increasing the number of biopsy procedures may require a longer time period and make patients more uncomfortable. In this study, it was found that the number of biopsy procedures in patients with positive cytological results was less than those with negative results $(2.9 \pm 1.3$ versus $3.7 \pm 1.8, \mathrm{p}=0.03)$. This implies that the biopsy procedure was stopped when imprint cytology was positive and proceeded when it was negative. With this approach, the number of biopsy procedures may be reduced 
in patients with malignant thoracic lesions without affecting accuracy of biopsy. Despite the fact that most of the patients (111 out of $127,87.4 \%$ ) with malignant thoracic lesions underwent one to four biopsy procedures, 16 patients required more than five biopsy procedures and 15 of them obtained a correct diagnosis. Without the aid of imprint cytology, false-negative results may have been encountered in these 15 patients.

This study has several limitations. First, it was a retrospective study. The imprint smear was selected and performed by the pulmonogist who conducted the procedure, which therefore led to a selection bias. Secondly, there was no on-site cytopathologist to assess the adequacy and diagnosis of the imprint smear. Although the smear was checked immediately by a pulmonologist, the tentative diagnoses could not be obtained in this retrospective study. Finally, there may be a discrepancy between the tentative diagnoses and the final diagnoses by a cytopathologist. Thus, a further prospective study with an on-site cytopathologist is needed to assess if imprint cytology for transthoracic core biopsy could reduce the number of biopsies and increase diagnostic accuracy.

In conclusion, imprint cytology for ultrasound-guided transthoracic core biopsy, which is a sensitive procedure for diagnosing malignant and benign thoracic lesions, may increase the diagnostic accuracy and cancer negative prediction. With an on-site approach, imprint cytology might assess the adequacy of biopsy specimens, optimise the biopsy procedure and provide a rapid diagnosis regarding the malignancy or benignity of a lesion. Since no significant amount of time, equipment or cost are needed to implement imprint cytology, this study advocates its use as part of the regular protocol for image-guided transthoracic core biopsy in this specified context.

\section{References}

1. Haramati LB. CT-guided automated needle biopsy of the chest. Am J Roentgenol 1995; 165: 53-55.

2. Kodama F, Ogawa T, Tanabe Y. Usefulness of CT-guided aspiration biopsy in combination with rapid cytology for diagnosis of benign pulmonary lesions. Nippon Igaku Hoshasen Gakkai Zasshi 1998; 58: 745-750.

3. Klein JS, Salomon G, Stewart EA. Transthoracic needle biopsy with a coaxially placed 20 -gauge automatic cutting needle: results in 122 patients. Radiology 1996; 198: 715-720.

4. Lucidarme O, Howarth N, Finet JF, Grenier PA. Intrapulmonary lesions: percutaneous automated biopsy with a detachable, 18-gauge, coaxial cutting needle. Radiology 1998; 207: 759-765.

5. Liao WY, Chen MZ, Chang YL, et al. US-guided transthoracic cutting biopsy for peripheral thoracic lesions less than $3 \mathrm{~cm}$ in diameter. Radiology 2000; 217: 685-691.

6. Sakai T, Hayashi N, Kimoto T, et al. CT-guided biopsy of the chest: usefulness of fine-needle core biopsy combined with frozen-section pathologic diagnosis. Radiology 1994; 190: $243-246$

7. Hayashi N, Sakai T, Kitagawa M, et al. CT-guided biopsy of pulmonary nodules less than $3 \mathrm{~cm}$ : usefulness of the springoperated core biopsy needle and frozen-section pathologic diagnosis. Am J Roentgenol 1998; 170: 329-331.

8. Dudgeon LS, Patrick CV. A new method for the rapid microscopical diagnosis of tumours: with an account of 200 cases so examined. Br J Surg 1927; 15: 250-261.

9. Suen KC, Wood WS, Syed AA, Quenville NF, Clement PB. Role of imprint cytology in intraoperative diagnosis value and limitations. J Clin Pathol 1978; 31: 328-337.

10. Owings RM. Rapid cytologic examination of surgical specimens: a valuable technique in the surgical pathology laboratory. Hum Pathol 1984; 15: 605-614.

11. Ghandur-Mnaymneh L, Paz L. The use of touch preparations (tissue imprints) in the rapid intraoperative diagnosis of metastatic lymph node disease in cancer staging procedures. Cancer 1985; 56: 339-344.

12. Clarke MR, Landreneau RJ, Borochovitz D. Intraoperative imprint cytology for evaluation of mediastinal lymphadenopathy. Ann Thorac Surg 1994; 57: 1206-1210.

13. Scucchi LF, Stefano DD, Cosentino L, Vecchione A. Value of cytology as an adjunctive intraoperative diagnostic method: an audit of 2250 consecutive cases. Acta Cytol 1997; 41: 1489-1496.

14. Hahn PF, Eisenberg PJ, Pitman MB, Gazelle GS, Mueller PR. Cytologic touch preparations (Imprints) form core needle biopsies: accuracy compared with that of fine-needle aspirates. Am J Roentgenol 1995; 165: 1277-1279.

15. Albert US, Duda V, Hadji P, et al. Imprint cytology of core needle biopsy specimens of breast lesions: a rapid approach to detecting malignancies, with comparison of cytologic and histopathologic analyses of 173 cases. Acta Cytol 2000; 44: 57-62.

16. Popp W, Rauscher H, Ritschka L, Redtenbacher S, Zwich H, Dutz W. Diagnosis sensitivity of different techniques in the diagnosis of lung tumours with the flexible fiberoptic bronchoscope: comparison of brush, imprint cytology of forceps biopsy, and histology of forceps biopsy. Cancer 1991; 67: 72-75.

17. Yang PC, Chang DB, Yu CJ, et al. Ultrasound-guided core biopsy of thoracic tumours. Am Rev Respir Dis 1992; 146: 763-767.

18. Yu CJ, Yang PC, Chang DB, et al. Evaluation of ultrasonically guided biopsy of mediastinal masses. Chest 1991; 100: 399-405.

19. Riu $\mathrm{CH}$. On the studies of the methods of staining blood film. J Nigada Med Assoc 1956; 70: 635-643.

20. Lee $\mathrm{CH}$, Wang $\mathrm{CH}$, Lin MC, et al. Multiple brushing with immediate Riu's stain via fiberoptic bronchoscopy without fluoroscopic guidance in the diagnosis of peripheral pulmonary tumours. Thorax 1995; 50: 18-21.

21. Lee CH, Liu CY, Wang CH, Ho YS, Chen TC. Use of Riu stain in the immediate interpretation of bronchial brushing cytology: comparison with Papanicolaou stain and histology. Acta Cytol 1997; 41: 1171-1177.

22. Cox JE, Chiles C, McManus CM, Aquino SL, Choplin RH. Transthoracic needle aspiration biopsy: variables that affect risk of pneumothorax. Radiology 1999; 212: 165-168.

23. Geraghty PR, Kee ST, McFarlane G, Razavi MK, Sze DY, Dake MD. CT-guided transthoracic aspiration biopsy of pulmonary nodules: needle size and pneumothorax rate Radiology 2003; 229: 475-481. 